2. O. Hanner, Retraction and extensions of mappings of metric and nonmetric spaces, Arkiv. för Mathematik, Astronomi och Fysik (1952) 315-360.

3. G. T. Whyburn, Analytic topology, Amer. Math. Soc. Colloquim Publications, vol. $28,1942$.

The University OF Wisconsin

\title{
SUBSETS OF AN ABSOLUTE RETRACT
}

\section{LINDA FALCAO FOULIS}

Introduction. In 1939 [6] Wojdyslawski asked whether the space $S(X)$ of subsets of an absolute retract $X$ is an absolute retract. This was answered in 1939 [6] for the case where $X$ is a bounded closed interval and in 1955 [2] for a Peano space. In this note the question is answered for all compact Hausdorff spaces. The author wishes to express her appreciation for the suggestions of Professor W. L. Strother.

Preliminaries. If $Y$ is a space then $S(Y)$ denotes the set of nonnull closed subsets of $Y$ with the usual topology [3]. (The results of this paper are valid for $S(Y)$ topologized in any other way in which the united extension defined in the following paragraph preserves continuity.) A compact space $Y$ is called a $C A R^{*}$ if every continuous function from a closed subset $M$ of a normal space $N$ to $Y$ can be extended to a continuous function from $N$ to $Y$. A definition for $M-C A R^{*}$ is obtained by replacing functions by multi-valued functions. The following are equivalent [5]: (1) $X$ is a $C A R^{*}$, (2) $X$ is homeomorphic to a retract of a Tychonoff cube, (3) every cube in which $X$ can be homeomorphically embedded as a closed subset $X_{0}$ can be retracted onto $X_{0}$.

A multi-valued function $F: X \rightarrow Y$ is said to be continuous at $x_{0}$ if (1) $V$ open and $F\left(x_{0}\right) \cap V \neq \varnothing$ implies that there is an open set $U$ containing $x_{0}$ such that, for all $x \in U, F(x) \cap V \neq \varnothing$ and (2) $V$ open and $F\left(x_{0}\right) \subset V$ implies that there is an open set $U$ containing $x_{0}$ such that $F(U) \subset V$. If $f: X \rightarrow Y$ is continuous then $f_{s}: S(X) \rightarrow S(X)$ defined by $f_{s}(A)=\bigcup\{f(a) \mid a \in A\}$ is called the united extension of $f$. $S_{1}(X)$ denotes $S(X)$ and $S_{n}(X)$ is defined inductively by $S_{n}(X)$ $=S\left[S_{n-1}(X)\right]$.

Received by the editors May 11, 1956. 


\section{Retracts.}

Lemma. If $T$ is a Tychonoff cube then $S(T)$ is a $C A R^{*}$.

Proof. The space $S(T)$ is compact Hausdorff and hence homeomorphic to a closed subset of some Tychonoff cube $R[1]$. The cubes $T$ and $R$ are, respectively, homeomorphic to subsets of $S(T)$ and $S(R)$. Identifying homeomorphic sets, one can write $T \subset S(T) \subset R$ $C S(R)$. Since $T$ is a $C A R^{*}$ contained in the cube $R$, there exists a retraction $p$ of $R$ onto $T$. If $f: X \rightarrow Y$ is continuous then the united extension $f_{s}: S(X) \rightarrow S(X)$ is continuous [2]. In case $f$ is a retraction of $X$ onto $Y$ it follows that $f_{s}$ is a retraction of $S(X)$ onto $S(Y)$. Hence corresponding to the retraction $p$ of $R$ onto $T$ there is a retraction $p_{s}$ of $S(R)$ onto $S(T)$. Then $p_{s}$ restricted to $R$ is a retraction of $R$ onto $S(T)$. This shows that $S(T)$ is homeomorphic to a retract of a cube $R$ and hence $S(T)$ is a $C A R^{*}$.

Theorem. If $X$ is a $C A R^{*}$ then $S(X)$ is a CAR*.

Proof. Embed $X$ in a cube $T$. Then $X$ is a retract of $T$ by definition of $C A R^{*}$. By united extension, $S(X)$ is a retract of $S(T)$. But $S(T)$ is a $C A R^{*}$ by the lemma, and $C A R^{*}$ is a retraction invariant [5]. Hence $S(X)$ is a $C A R^{*}$.

Corollary 1. Every $C A R^{*}$ is an $M-C A R^{*}$.

Proof. It is known [2] that $X$ is an $M-C A R^{*}$ if and only if $S(X)$ is a $C A R^{*}$.

Corollary 2. If $X$ is a CAR* then $S_{n}(X)$ is a CAR*.

This theorem also provides a new proof for the theorem that the space of subsets of a $C A R^{*}$ has the fixed point property [4]. Whether $X$ having the fixed point property implies that $S(X)$ has the fixed point property remains open.

\section{REFERENCES}

1. J. L. Kelly, General topology, New York, Van Nostrand, 1955.

2. W. L. Strother, Fixed points, fixed sets, and M-retracts, Duke Math. J. vol. 22 (1955) pp. 551-556.

3. — Multi-homotopy, Duke Math. J. vol. 22 (1955) pp. 281-286.

4. C. E. Capel and W. L. Strother, $A$ space of subsets having the fixed point property, Proc. Amer. Math. Soc. vol. 7 (1956) pp. 707-708.

5. A. D. Wallace, Outline for algebraic topology, New Orleans, 1950.

6. M. Wojdyslawski, Retractes absolus et hyperespaces des continus, Fund. Math. vol. 32 (1939) pp. 184-192.

UNIVERSITY OF MiAMI 